\title{
Quantitative Analysis of Vaginal Wall Thickness in Vaginal Cuff Brachytherapy - Multichannel Balloon vs. Single-Channel Brachytherapy
}

\author{
Junfang Yan', An Liu', Scott Glaser², Iris Liang', Fuquan Zhang', Jeffrey Wong ${ }^{2}$ and Yi-Jen \\ Chen $^{2 *}$
}

${ }^{1}$ Department of Radiation Oncology, Peking Union Medical College Hospital, China

${ }^{2}$ Department of Radiation Oncology, City of Hope Medical Center, USA

*Corresponding author: Yi-Jen Chen, Department of Radiation Oncology, City of Hope Medical Center, 1500 E Duarte Road, Duarte, CA 91010, USA.

To Cite This Article: Iris Liang, Fuquan Zhang, Jeffrey Wong, Yi-Jen Chen. Quantitative Analysis of Vaginal Wall Thickness in Vaginal Cuff Brachytherapy - Multichannel Balloon vs. Single-Channel Brachytherapy. A Review. Am J Biomed Sci \& Res. 2021 - 11(6). AJBSR.MS.ID.001684. DOI: 10.34297/AJBSR.2021.11.001684.

Received: 阱 January 14, 2020; Published: 眥 February 03, 2021

\begin{abstract}
Objective: To evaluate thickness of the proximal vaginal wall during vaginal cuff brachytherapy (VCB) and to compare results between cases treated using single-channel cylinder (SCC) versus multichannel balloon (MCB).

Methods: A total of 82 consecutive cases, including 41 by SCC and 41 by MCB treatment, were reviewed. Vaginal wall thickness was measured using CT images captured during VCB. Multiple points along the proximal vaginal canal were measured, including the central apex position on the top of vagina and the anterior, posterior, left, and right lateral positions at planes $0.5,1$, 2, and 3-cm from the top of vagina. Independent sample's t-tests and Wilcoxon Mann Whitney tests were used for statistical analysis.

Results: The thickness of proximal vaginal wall varied significantly at different locations (apex, lateral, anterior, and posterior). The mean thickness at the apex was 0.51 and $0.50 \mathrm{~cm}$ for SCC and MCB cases respectively $(\mathrm{p}=0.391)$. The vaginal wall was much thicker at both left and right lateral positions compared to anterior and posterior positions at all measurement planes for both SCC and MCB VCB. The mean vaginal wall thickness at lateral positions exceeded $0.5 \mathrm{~cm}$ for all measurement planes for SCC. In contrast, the mean thickness at lateral positions was smaller than $0.5 \mathrm{~cm}$ in all planes except measurements done at 0.5 and $1-\mathrm{cm}$ distance from the apex for MCB.
\end{abstract}

Conclusion: Compared to SCC, MCB can stretch and thin the vaginal wall, especially at the lateral directions and provide dosimetric advantages.

Keywords: Vaginal wall thickness; Vaginal cuff brachytherapy; Single-channel cylinder; Multichannel balloon

Abbreviations: VCB: Vaginal Cuff Brachytherapy; SCC: Single-Channel Cylinder; MCB: Multichannel Balloon; EBRT: External Beam Radiotherapy; PORTEC: Post-Operative Radiation Therapy in Endometrial Carcinoma; ABS: American Brachytherapy Society; CT: Computerized Tomography; MRI: Magnetic Resonance Imaging; SPSS: Statistical Package for the Social Sciences; DVH: Dose-Volume Histogram

\section{Introduction}

Cancers of the uterine corpus and cancers of the cervix represent the first and the third most common gynecologic malignancies in the United States, respectively. In 2019, 61,880 new diagnoses and 12,160 deaths are estimated to be due to uterine cancer [1]. Similarly, 13,170 new diagnoses and 4,250 deaths are estimated to be due to cervical cancer [1]. Surgery is the primary management for uterine corpus cancer and early-staged cervical cancer. Postoperatively, adjuvant vaginal cuff brachytherapy (VCB) alone or in combination with external beam radiotherapy (EBRT) has become an integral treatment to reduce risk of loco-regional disease recurrence.

The National Comprehensive Cancer Network recommends postoperative adjuvant EBRT and/or VCB for endometrial cancer based on risk factor assessment including age, grade, depth of 
myometrial invasion, and lymphovascular invasion [2]. Supported by the results of the PORTEC- 2 trial, postoperative VCB alone is considered a reasonable recommendation for patients with stage I endometrial cancer with high-intermediate risk [2,3]. There is no clear agreement regarding the indications of VCB for postoperative cervical cancer. Consensus guidelines from the American Brachytherapy Society (ABS) recommend using VCB for patients who have less than radical hysterectomy or who have close or positive resection margins, large or deeply invasive tumors, parametrial or vaginal involvement, or extensive lymphovascular invasion $[4,5]$.

In general, VCB is used to cover the proximal $3-5 \mathrm{~cm}$ of the vagina, and the dose is prescribed to either the vaginal surface or $0.5-\mathrm{cm}$ depth [4]. However, little is known about the volume of tissue superior to the vaginal cuff and the thickness of proximal vaginal canal. Therefore, the true dosimetry to the target volume in vaginal cuff is not clear [6]

We have adopted and used multi-channel balloons (MCBs; Capri applicator, Varian Medical Systems, Inc., Palo Alto, CA) for VCB since June 2014. Compared to single-channel cylinders (SCCs), we found that MCB can cover a larger volume and deliver a more conformal and homogeneous target coverage [7]. Hypothesizing there is high variability of proximal vaginal wall thickness, we sought to evaluate and compare the thickness of the proximal vaginal wall during VCB by SCC versus MCB.

\section{Materials and Methods}

With institutional review board approval, the medical records of 82 consecutive patients treated with postoperative high-dose-rate VCB between January 2011 and December 2018 were reviewed. There were 65 endometrial cancer and 17 cervical cancer patients. Details of VCB using either SCC or MCB were described in our previous paper [7]. Briefly, all patients underwent either VCB alone or a combination of EBRT and VCB after surgery. Patients were instructed to empty their rectum and bladder before VCB. Each patient underwent a pelvic examination to ensure adequate healing of the vaginal cuff and assess the size and length of vaginal canal. For cases before June 2014, a SCC (CT and MRI compatible vaginal cylinder, GM11004140; Varian Medical System, Inc., Palo Alto CA) with variable sizes was used. In general, the largest diameter SCC that the patient could comfortably accommodate was selected for treatment. For cases after June 2014, an MCB was preferentially used. The deflated MCB was inserted up to the vaginal apex and inflated with air to adapt to the patient's vaginal anatomy. The inflation stopped when the patient felt and expressed pressure discomfort. The applicator was then secured in place by a T-binder for RT planning and treatment. Positions of the MCB were verified by CT scanning with $2.5-\mathrm{mm}$ slice thickness. The CT studies were transferred to the Varian Eclipse Treatment Planning System (Varian Medical Systems, Palo Alto, CA) for RT planning. For cases treated by VCB alone, the prescription was 7 Gy to 5-mm depth from vaginal mucosal surface covering proximal $3-\mathrm{cm}$ vagina. The treatment was done on a weekly basis up to a total of 3 treatments. For cases treated by combined EBRT and VCB, VCB was done after completion of EBRT and the prescription dose was reduced to $4 \mathrm{~Gy}$ per fraction up to 3 treatments in 2 weeks. For the purpose of this study, only the CT planning images of the first fraction of VCB for each patient were evaluated.

The following protocol was developed to define the proximal vaginal wall thickness: (1) the window level of the CT images was consistently set at the pre-defined "pelvis" in the Varian Eclipse Treatment Planning System; (2) images of the sagittal and coronal planes were reconstructed along the central axis of the applicators; (3) the vaginal wall thickness perpendicular to the vaginal apex along the central axis was measured; (4) subsequently, the vaginal wall thickness perpendicular to the surface of the vaginal applicators at planes $0.5,1,2$, and 3 -cm from the top of vagina along the central axis were measured on both the sagittal and coronal planes, and these represented anterior, posterior, left lateral, and right lateral positions of the vaginal canal (Figure 1).
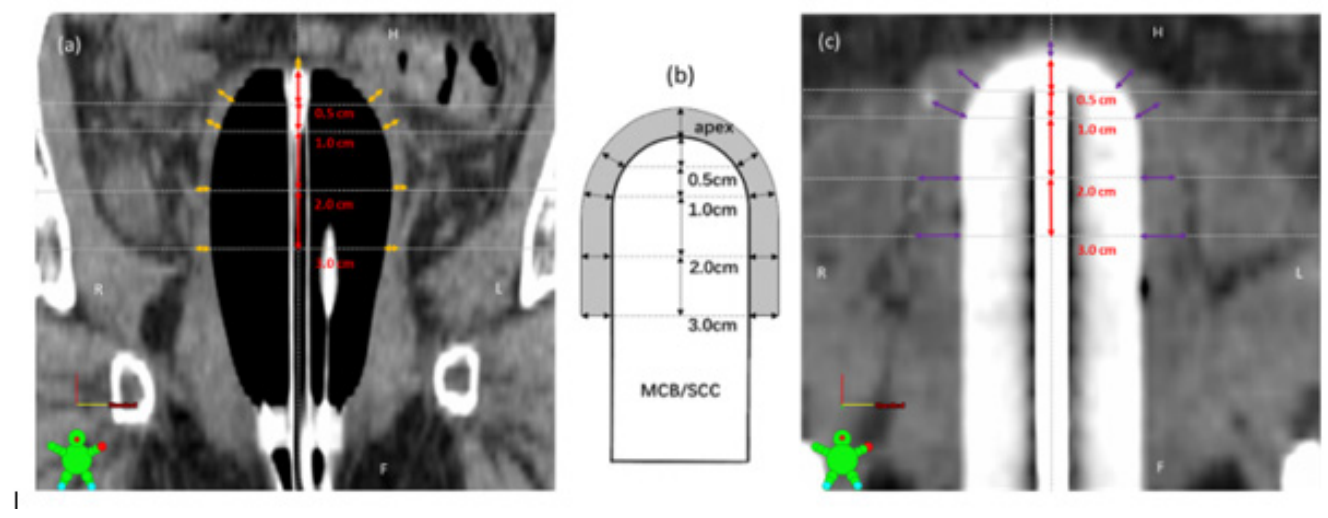

Figure 1: Measurement of proximal vaginal wall thickness during VCB; a. coronal view by MCB, b. schematic illustration of vaginal wall thickness measurement, c. coronal view by SCC. 
Independent sample's t-tests were used to compare the vaginal wall thickness at different positions and between SCC versus MCB treatments. The Wilcoxon Mann Whitney tests (nonparametric simulated) were used when the dependent variables were not normally distributed interval variables. The chi-squared test was used to determine whether there was a significant difference between frequencies of two groups. Statistical analyses were performed with SPSS 22.0, and two-tailed p-Values lower than 0.05 were considered to be significant.

\section{Results}

Table 1 shows the characteristics of patients treated with SCC and MCB. Forty-one patients, including 15 cervical cancers and 26 endometrial cancers, were treated with SCC applicators and another 41 patients, including 4 cervical cancers and 37 endometrial cancers, were treated with MCB applicators. A higher proportion of patients in the MCB group received VCB alone compared to patients in the SCC group (39.0\% vs. 19.5\%). The average size of SCC used was $2.9-\mathrm{cm}$ diameter (range, $2.3-3.5 \mathrm{~cm}$ ) and the average amount of air inflated in MCB during VCB was 25.2 cc (range, 5-50 cc).

The mean and range of the thickness at vaginal apex were similar for both treatment groups, $0.51(0.26-1.76) \mathrm{cm}$ and 0.50 $(0.17-1.62) \mathrm{cm}$ for SCC and MCB groups, respectively ( $\mathrm{p}=0.391)$. However, in 22 of 41 patients by SCC (53.7\%) and 18 of 41 cases by
MCB (43.9\%), the vaginal wall thickness at apex exceeded $0.5 \mathrm{~cm}$ implying potential under dose in a significant number of patients at the vaginal apex if the prescription point is placed at $0.5 \mathrm{~cm}$ depth from the top of vaginal mucosa.

Table 2 shows results of the thickness of the proximal vaginal wall at planes $0.5,1,2$, and $3 \mathrm{~cm}$ from the top of vagina by SCC and MCB applicators. The thickness varied significantly between left and right lateral positions versus anterior and posterior positions in both SCC and MCB groups. The vaginal walls were much thicker at both left and right lateral positions compared to anterior and posterior positions at all measurement planes for both SCC and MCB groups. In addition, the mean vaginal wall thickness exceeded $0.5 \mathrm{~cm}$ at left and right lateral positions for all measurement planes using SCC applicators. Specifically, 198 out of a total of 328 measurements (60.4\%) over lateral positions by SCC exceeded 0.5 $\mathrm{cm}$, and 10 measurements exceeded $1.0 \mathrm{~cm}$. In contrast, the mean vaginal wall thickness by MCB applicators at lateral positions was smaller than $0.5 \mathrm{~cm}$ in all planes except measurements done at $0.5 \mathrm{~cm}$ (both sides) and $1 \mathrm{~cm}$ (left side only) from the apex. The mean vaginal wall thickness by MCB applicators at lateral positions reduces gradually towards distal end. However, 79 out of a total of 328 measurements $(24.1 \%)$ over lateral positions by MCB exceeded $0.5 \mathrm{~cm}$, and there were 4 measurements over $1.0 \mathrm{~cm}$.

Table 1: Characteristics of patients treated with SCC and MCB.

\begin{tabular}{|l|c|c|}
\hline \multicolumn{1}{|c|}{ Characteristics } & SCC & \multicolumn{2}{|c|}{ MCB } \\
\hline No. of patients & 41 & 4 \\
\hline Diagnosis & 15 & 37 \\
\hline Cervical cancer & 26 & 30.4 \\
\hline Endometrial cancer & 55.0 & $30.84 \pm 7.44$ \\
\hline Age (mean) & $28.77 \pm 8.22$ & $16(39.0 \%)$ \\
\hline BMI (mean \pm SD) & & $25(61.0 \%)$ \\
\hline Treatment & $8(19.5 \%)$ & N/A \\
\hline VCB (\%) & $33(80.5 \%)$ & $25.2 \mathrm{cc}(5-50 \mathrm{cc})$ \\
\hline EBRT + VCB (\%) & $2.9 \mathrm{~cm}(2.3-3.5 \mathrm{~cm})$ & \multicolumn{2}{|c|}{} \\
\hline Diameter of SCC (mean; range) & N/A & \\
\hline Air inflated in MCB (mean; range)
\end{tabular}

SCC: Single-Channel Cylinder; MCB: Multichannel Balloon; BMI: Body Mass Index; N/A: Not Applicable

Table 2: Vaginal wall thickness at planes $0.5,1,2$ and $3 \mathrm{~cm}$ from the top of vagina by SCC and MCB applicators.

\begin{tabular}{|c|c|c|c|c|c|c|c|c|c|}
\hline \multicolumn{2}{|c|}{ Plane level (cm) } & \multicolumn{2}{|c|}{0.5} & \multicolumn{2}{|c|}{1} & \multicolumn{2}{|c|}{2} & \multicolumn{2}{|c|}{3} \\
\hline & & Mean \pm SD & Range & Mean \pm SD & Range & Mean \pm SD & Range & Mean \pm SD & Range \\
\hline \multirow{3}{*}{$\begin{array}{c}\text { Anterior } \\
(\mathrm{cm})\end{array}$} & SCC & $0.50(0.16)$ & $0.25-1.08$ & $0.48(0.18)$ & $0.20-1.20$ & $0.45(0.12)$ & $0.19-0.70$ & $0.49(0.10)$ & $\begin{array}{c}0.28- \\
0.72\end{array}$ \\
\hline & МCB & $0.42(0.14)$ & $0.23-0.86$ & $0.35(0.09)$ & $0.21-0.59$ & $0.36(0.09)$ & $0.19-0.55$ & $0.35(0.07)$ & $\begin{array}{c}0.23- \\
0.50\end{array}$ \\
\hline & $\mathrm{p}$ & \multicolumn{2}{|c|}{0.006} & \multicolumn{2}{|c|}{0.000} & \multicolumn{2}{|c|}{0.000} & \multicolumn{2}{|c|}{0.000} \\
\hline
\end{tabular}




\begin{tabular}{|c|c|c|c|c|c|c|c|c|c|}
\hline \multirow{3}{*}{$\begin{array}{c}\text { Posterior } \\
\text { (cm) }\end{array}$} & SCC & $0.36(0.12)$ & $0.16-0.70$ & $0.34(0.13)$ & $0.17-0.89$ & $0.32(0.07)$ & $0.14-0.45$ & $0.33(0.09)$ & $\begin{array}{c}0.18- \\
0.61\end{array}$ \\
\hline & МCB & $0.26(0.11)$ & $0.10-0.70$ & $0.22(0.09)$ & $0.10-0.67$ & $0.25(0.08)$ & $0.10-0.45$ & $0.25(0.07)$ & $\begin{array}{c}0.10- \\
0.42\end{array}$ \\
\hline & $\mathrm{p}$ & \multicolumn{2}{|c|}{0.000} & \multicolumn{2}{|c|}{0.000} & \multicolumn{2}{|c|}{0.000} & \multicolumn{2}{|c|}{0.000} \\
\hline \multirow{3}{*}{$\begin{array}{l}\text { Right lateral } \\
\qquad(\mathrm{cm})\end{array}$} & SCC & $0.58(0.23)$ & $0.30-1.40$ & $0.58(0.25)$ & $0.23-1.32$ & $0.56(0.18)$ & $0.24-1.03$ & $0.56(0.19)$ & $\begin{array}{c}0.23- \\
0.94\end{array}$ \\
\hline & МCB & $0.53(0.21)$ & $0.27-1.06$ & $0.44(0.11)$ & $0.24-0.73$ & $0.40(0.09)$ & $0.19-0.67$ & $0.37(0.09)$ & $\begin{array}{c}0.19- \\
0.52\end{array}$ \\
\hline & $\mathrm{p}$ & \multicolumn{2}{|c|}{0.189} & \multicolumn{2}{|c|}{0.005} & \multicolumn{2}{|c|}{0.000} & \multicolumn{2}{|c|}{0.000} \\
\hline \multirow{3}{*}{$\begin{array}{l}\text { Left lateral } \\
\qquad(\mathrm{cm})\end{array}$} & SCC & $0.52(0.19)$ & $0.20-1.24$ & $0.58(0.16)$ & $0.35-1.00$ & $0.63(0.21)$ & $0.37-1.33$ & $0.61(0.14)$ & $\begin{array}{c}0.37- \\
0.90\end{array}$ \\
\hline & MCB & $0.58(0.30)$ & $0.23-1.83$ & $0.52(0.17)$ & $0.23-0.98$ & $0.41(0.11)$ & $0.12-0.78$ & $0.39(0.10)$ & $\begin{array}{c}0.22- \\
0.62\end{array}$ \\
\hline & $\mathrm{p}$ & \multicolumn{2}{|c|}{0.707} & \multicolumn{2}{|c|}{0.103} & \multicolumn{2}{|c|}{0.000} & \multicolumn{2}{|c|}{0.000} \\
\hline
\end{tabular}

SCC: Single-Channel Cylinder; MCB: Multichannel Balloon; cm: Centimeter

The mean vaginal wall thickness at anterior and posterior positions for both types of applicators was equal to or less than 0.5 $\mathrm{cm}$ in all measurement planes. Compared to the results by SCC, the mean vaginal wall thickness by MCB at either anterior or posterior positions was significantly smaller in all measurement planes.

\section{Discussion}

This is the first study to measure thickness of the proximal vaginal wall during VCB. We found that the thickness of the vaginal wall varied significantly at different locations. In approximately $50 \%$ of patients, the vaginal wall thickness at apex exceeded 0.5 $\mathrm{cm}$. In addition, the vaginal wall was thicker at both left and right lateral positions compared to anterior and posterior positions. Compared to SCC, MCB opens and stretches the vaginal canal more and possibly provides better dosimetric coverage.

The goal of post-operative adjuvant VCB is to reduce local cancer recurrence. In general, the proximal vaginal canal, especially the vaginal apex, is the target of VCB. Effective VCB treatment regimens vary significantly between major institutions regarding target length coverage, depth of dose specification, dose per fraction, and total dose [8]. A pathologic study by Choo et al. demonstrated that approximately $95 \%$ of vaginal lymphatic channels were located within 3-mm depth from the vaginal surface. Therefore, VCB dose prescribed to a depth of less than $5 \mathrm{~mm}$ might be adequate. ABS recommends treating the proximal $3-5 \mathrm{~cm}$ of vagina and that the dose be specified at either the vaginal surface or $0.5-\mathrm{cm}$ depth [4]. Based on the PORTEC 2, the most commonly used regimen for VCB alone is 7 Gy $\times 3$ prescribed to $0.5-\mathrm{cm}$ depth from the vaginal mucosal surface [3]. A lower dose prescribed to the vaginal surface, such as 6 Gy x 5 or 4 Gy x 6, may be effective as well. Consistent with this, a randomized study by Sorbe et al. [9] comparing 2.5 Gy/fraction vs. $5.0 \mathrm{~Gy} /$ fraction $\mathrm{x} 6$ prescribed to $0.5 \mathrm{~cm}$ depth had similar oncologic outcomes, with only one vaginal recurrence in each arm of the study. In addition, at the 5-year follow up, cases treated with higher dose per fraction had higher degrees of vaginal shortening, mucosal atrophy, and bleeding.

The ABS recommends using the largest diameter cylinder that can comfortably and snugly fit the vagina for treatment [4]. However, due to the wider proximal end and the high-pressure constraint over vaginal introitus [10-13], it is often difficult to insert an adequately sized cylinder so that it fits snugly to the vagina apex. As illustrated by the current study, in cases treated by a rigid SCC, although the mean thickness of anterior and posterior wall were all equal to or less than $0.5 \mathrm{~cm}$, the mean thickness of vaginal wall at lateral aspects was not stretched well enough, and most were more than $0.5 \mathrm{~cm}$. This indicates a single-channel applicator with symmetric cylindrical configuration is not an ideal applicator for this type of treatment. With inflatable balloon and multi-channel capability, MCB can provide more conformal and homogeneous target coverage [7]. From the current study, compared to the results by SCC, the mean vaginal wall thickness by MCB were significantly smaller in all measurement planes except lateral aspects at $0.5-\mathrm{cm}$ distance from the apex and 1-cm distance from the apex on the left side. With MCB applicators, the thickness at $0.5-\mathrm{cm}$ distance on left and right directions and $1-\mathrm{cm}$ distance from the apex on the left side were still more than $0.5 \mathrm{~cm}$. This is likely related to the design of MCB applicator, which tends inflate easier in the middle compared to both ends. Future studies should focus on designing balloon applicators adapting to postoperative contour of the proximal vaginal canal for a better dosimetric coverage.

The mean thickness of vaginal wall over anterior and posterior aspects was all less than $0.5 \mathrm{~cm}$ in all measurement planes by either applicator. Specifically, the mean thickness of vaginal wall at the posterior aspects was less than $0.3 \mathrm{~cm}$ in all measurement planes by MCB. Therefore, using MCB, it is essential to evaluate the thickness of the vaginal wall and optimize the VCB plan by adjusting the dwell time weighting on peripheral channels to reduce unnecessary 
radiation exposure to the bladder and/or rectum. Indeed, Onsrud et al. [14] found less late vaginal and bladder reactions with individualized treatment plans by reducing prescription depth point from $5 \mathrm{~mm}$ to 3,4 or $5 \mathrm{~mm}$ based on mucosal thickness. Kim et al. [15] reported that significant dose reduction to rectum and bladder could be achieved without compromising target coverage by customizing the VCB dosimetry based on individual patient anatomy by using 3-dimensional RT planning. A dosimetric study on MCB by Park et al. found dose reduction to bladder, rectum, sigmoid, and urethra when using all 13 channels compared to results using a single central channel alone. With $5 \mathrm{~mm}$ depth prescription, the absolute reduction of D2cc of rectum and bladder was $14.6 \%$ and $9.0 \%$ of the prescription dose, respectively. In addition, the mean dose reduction to rectum and bladder was $6.4 \%$ and $4.3 \%$, respectively [16].

Limitations of the present study include a retrospective study design, limited case number, imbalanced patient population, lack of clinical outcome data, and lower soft tissue image resolution by CT scan compared to MRI study [6]. The MCB group had significantly older patients (mean age 60.4 versus 55.0) and higher proportion of endometrial cancer patients (90\% versus 63\%). One can argue women of older age and endometrial diagnosis might have significantly thinner/more atrophic vaginal mucosa than younger/ cervical cancer patients. Furthermore, the MCB group had fewer combined modalities as opposed to VCB alone patients $(61.0 \%$ verse $80.5 \%$ ), and it is possible that the vaginal thickness is greater in patients that received combined treatment over those treated with VCB alone due to inflammation/fibrosis. It is well known fact that the measurement of vaginal wall thickness is subjective and highly depends on individual doing the management. Therefore, further investigation will be needed to confirm the results of the current study.

In the future we plan to compare the results between a more balanced group of patients and to use MRI by dose-volume histogram (DVH)-based metrics to evaluate the actual benefit between the two types of applicators.

\section{Conclusion}

There is high variability in the thickness of proximal vaginal wall. Compared to SCC, MCB can better stretch and thin the vaginal wall, especially laterally, and can provide dosimetric advantages. In addition, MCB offers multiple dwell channels and positions to optimize the dose distribution. To further improve the dosimetric coverage, future studies should focus on improving the inflatable multi-channel balloon to adapt to the contour of the proximal vagina and to improve opening of the lateral aspects of the vagina.

\section{Acknowledgement}

The authors thank Chris Gandhi from the Office of Faculty and Institutional Support of City of Hope for his edits and comments on the manuscript. The study is supported by PUMCH Faculty Development Overseas Training Program (PUMCHFD).

\section{Conflict of Interest}

All the authors report no proprietary or commercial interest in any product mentioned or concept discussed in this article.

\section{References}

1. Siegel RL, Miller KD, Jemal A (2019) Cancer statistics, 2019. CA Cancer J Clin 69(1): 7-34.

2. Koh WJ, Abu-Rustum NR, Bean S, Bradley K, Campos SM, et al. (2018) Uterine neoplasms, version 1.2018 , clinical practice guidelines in oncology. JNCCN 16(2): 170-199.

3. Nout RA, Smit VT, Putter H, Jurgenliemk-Schulz IM, Jobsen JJ, et al. (2010) Vaginal brachytherapy versus pelvic external beam radiotherapy for patients with endometrial cancer of high-intermediate risk (PORTEC-2): an open-label, non-inferiority, randomised trial. Lancet 375(9717): 816823.

4. Small W Jr, Beriwal S, Demanes DJ, Dusenbery KE, Eifel P, et al. (2012) American Brachytherapy Society consensus guidelines for adjuvant vaginal cuff brachytherapy after hysterectomy. Brachytherapy 11(1): 58-67.

5. Li R, Shinde A, Chen Y, Amini A, Lee S, et al. (2018) Survival benefit of adjuvant brachytherapy after hysterectomy with positive surgical margins in cervical cancer. Int J Radiat Oncol Biol Phys 102(2): 373-382.

6. Chapman CH, Prisciandaro JI, Maturen KE, Cao Y, Balter JM, et al. (2016) MRI-based evaluation of the vaginal cuff in brachytherapy planning: are we missing the target? Int J Radiat Oncol Biol Phys 95(2): 743-750.

7. Hou X, Liu A, Zhang F, Wong J, Chen Y (2016) Dosimetric advantages of using multichannel balloons compared to single-channel cylinders for high-dose-rate vaginal cuff brachytherapy. Brachytherapy 15(4): 471476.

8. Harkenrider MM, Block AM, Alektiar KM, Gaffney DK, Jones E, et al. (2017) American Brachytherapy Task Group Report: Adjuvant vaginal brachytherapy for early-stage endometrial cancer: A comprehensive review. Brachytherapy 16(1): 95-108.

9. Sorbe B, Straumtis A, Karlsson L (2005) Intravaginal high-dose-rate brachytherapy for stage I endometrial cancer: A randomized study of two dose-per-fraction levels. Int J Radiat Oncol Biol Phys 62(5): 13851389.

10. DeLancey JO (1992) Anatomic aspects of vaginal eversion after hysterectomy. Am J Obstet Gynecol 166(6): 1717-1724.

11. Ramanah R, Berger MB, Parratte BM, DeLancey JOL (2012) Anatomy and histology of apical support: a literature review concerning cardinal and uterosacral ligaments. Int Urogynecol J 23(11): 1483-1494.

12. Guaderrama NM, Nager CW, Liu J, Pretorius DH, Mittal RK (2005) The vaginal pressure profile. Neurourol Urodyn 24(3): 243-247.

13. Luo J, Betschart C, Ashton-Miller JA, DeLancey JOL (2016) Quantitative analyses of variability in normal vaginal shape and dimension on MR images. Int Urogynecol J 27(7): 1087-1095.

14. Onsrud M, Strickert T, Marthinsen ABL (2001) Late reactions after postoperative high-dose-rate intravaginal brachytherapy for endometrial cancer: a comparison of standardized and individualized target volumes. Int J Radiat Oncol Biol Phys 49(3): 749-755.

15. Kim H, Kim H, Houser C, Beriwal S (2012) Is there any advantage to threedimensional planning for vaginal cuff brachytherapy? Brachytherapy 11(5): 398-401.

16. Park SJ, Chung M, Demanes DJ, Banerjee R, Steinberg M, et al. (2013) Dosimetric comparison of 3-dimensional planning techniques using an intravaginal multichannel balloon applicator for high-dose-rate gynecologic brachytherapy. Int J Radiat Oncol Biol Phys 87(4): 840-846. 